\title{
Cause and effects of global cooling
}

\section{by John Gribbin}

A RECENT flurry of papers has provided further evidence for the belief that the Earth is cooling. There now seems little doubt that changes over the past few years are more than a minor statistical fluctuation.

Last year, Kukla and Kukla (Science, 183,709 ; 1974) reported satellite observations of the sudden growth of ice cover in the northern hemisphere in the winter of 1971-72, and suggested that the increase was equivalent to onesixth of the change needed to bring about a full ice age. On page 45 of this issue of Nature, Wahl and Bryson compare recent sea surface temperature patterns with those of cooler regimes in the past, and conclude that over the period from 1951 to 1972 there was a decline corresponding "to a return of about one-sixth of the way to a full ice age". It is tempting to suggest that the change in sea surface temperatures contributed in some way to the spread of ice noted by Kukla and Kukla.

But there is as yet no evidence that further cooling is likely in the immediate future. The observed cooling corresponds to a re-establishment of the 'Little Ice Age' which persisted for several hundred years up to the end of the nineteenth century; it may be that all that has happened since 1950 is that the unusually mild spell of the first part of this century has ended. Over the past millenium, icier conditions than those of today have been the norm. Indeed, ice ages have been common over the past two million years. But on longer time scales still, such cool phases are relatively rare events-and there is now a plausible model to explain why this should have been so.

At present, the land surface of the Earth is concentrated in the northern hemisphere, around the almost landlocked polar sea. This is a situation which can encourage the spread of ice much more than if warm water could easily penetrate to the polar sea, although of course polar ice forms even more easily when the pole is covered by land, as in Antarctica today. It seems that over the past couple of million years this distribution of the continents has resulted in a fairly delicate balance between ice ages and interglacial regimes. If ice cover is established, its high albedo is enough to maintain glacial conditions for a long time; but once the ice has gone a definite trigger (such as volcanic activity or changes in the Earth's orbit; see page 20) is needed to re-establish it.

Calculations presented by Sellers and Meadows (on page 44 of this issue) support the commonly held view that the present-day distribution of northern continents may be the root cause of the wave of ice ages in the past two million years. Neglecting other effects, if the continents were gathered in a belt around the equator, Sellers and Meadows find that the difference in albedo from conditions like those of the present would raise the global mean surface temperature by $12^{\circ} \mathrm{C}$.

Much more work of this kind needs to be done before plate tectonics can provide a full insight into the long-term changes in patterns of glaciation, but at least the link between continental drift and ice ages is now beginning to be put on a quantitative basis.

\section{Eukaryotic operon}

\section{from P. J. Ford}

Genetic regulatory systems in prokaryotes are quite well understood compared with those in eukaryotes. Regulator genes code for regulator substances (usually proteins in prokaryotes) which function by interacting with regulator sites (operators) in the DNA adjacent to the structural genes under control and with small molecules (either inducers which are usually substrates or repressors which are usually end products) which alter their affinity for the regulator sites. A simple model supposes that regulator substances exist in two forms, one capable and one incapable of binding to the regulator site. Inducers and repressors shift the equilibrium between these two forms.

Four types of gene control circuit are theoretically possible using these components. Negative control exists when the binding of regulator protein (repressor) at the regulator site inhibits gene transcription. In this case gene activity may be restored (induced) by inactivation of the repressor in the presence of inducer or eliminated (repressed) by activation of the repressor protein in the presence of co-repressor substance which is essential for repressor activity. Positive control exists when binding of regulator protein (inducer protein) at the regulator site is essential for gene transcription. Inducible positive control is found when the presence of an effector substance (coinducer), as well as inducer protein, is required for gene activity and repressible positive control occurs when the co-repressor inactivates the inducer protein, eliminating gene activity.

Regulator gene mutants in negative control circuits must be recessive if structural gene function occurs in the absence of inducer (constitutive) or in the presence of co-repressor (derepressed); or dominant if there is no expression in the presence of inducer (uninducible) or in the absence of repressor (super-repressed). In positive control circuits the dominance and recessive relationships are reversed. Mutations in regulator sites are always dominant when linked (cis configuration) to structural genes and recessive when unlinked (trans configuration). Mutations in regulator sites of negative control circuits are constitutive or derepressed and of positive control circuits are uninducible or su perrepressed.

These four theoretically possible types of simple control circuit are not equally used. Most control circuits in prokaryotes are under negative control and either inducible, like the lactose operon, or repressible, like the tryptophan operon. An inducible positive control system is the cyclic AMP system which controls several inducible operons (galactose for instance) and such operons are under dual control. In this system cyclic AMP, which is neither a substrate for, nor a product of the enzyme pathway, activates a binding protein which is required for transcription of the operon. A further complication may arise when a regulator protein acts both in a negative and a positive way such as is found in the control of the arabinose operon. In this circuit the regulator protein acts as a repressor in the absence of arabinose but in its presence binds to an initiator site, allowing gene activity.

A second type of control site (called promoter or polymerase binding site) which may be completely separate from or partially overlap the operator site has been found in many prokaryotic control circuits. Promoter sites are usually located distal to negative and proximal to positive control operators. Theoretically, positive control may be exercised by interaction of control proteins (sigma factors) with the polymerase core enzyme altering its specificity towards promoter sequences. Mutations in promoter sequences will 